greater habit strength (H) than 15 trials. At the same time. $r_{R}$ might be expected to have reached asymptote by 15 multiple-pellet trials. It is possible, then, to compare the single and multiple groups after 15 trials as having similar levels of $H$ with the multiple group having greater $r_{R}$. Likewise, the 15-multiple-pellet group may be taken to have a similar level of $t_{R}$ as the 60-multiple-pellet group, but less $\mathrm{H}$. Given these reasonable assumptions, it can be concluded that with $I_{R}$ equated, resistance to extinction will increase with $H$, but with $\mathrm{H}$ equated resistance to extinction will decrease with $r_{R}$. These conclusions are exactly those of Theios \& Brelsford (1964), who provided goalbox placements to increase the strength of $r_{R}$ while maintaining control over the growth of $H$. Goalbox placements are probably very similar to the multiple-pellet trials of the present experiment in that both appear to augment $r_{R}$ while not substantially contributing to $H$. Thus, the evidence seems to favor the Trapold interpretation, that $r_{R}$ can grow with approximations of the complete instrumental response.

It may also be noted that the present experiment found no extinction differences for the pellet groups following five training trials. Amsel, Hug, \& Surridge (1968), on the other hand, demonstrated greater resistance to extinction following five trials when their Ss were given two pellets than when given 24 pellets. There are numerous procedural differences between the present experiment and that of Amsel et al, but perhaps the one most relevant to the dissimilarity in extinction is the reward magnitude employed. Amsel et al used a reward twice the size $(1,000 \mathrm{mg})$ as that used in the present study $(500 \mathrm{mg})$. With the larger reward, $\mathrm{r}_{\mathrm{R}}$ should condition more rapidly; thus any differences due to $r_{R}$ should tend to occur earlier in acquisition. It is not contradictory, then, that the more rapid extinction of the multiple-pellet group following 15 training trials observed in the present experiment was seen in the Amsel et al experiment following 5 training trials.

\section{REFERENCES}

AMSEL, A. Partial reinforcement effects of vigor and persistence. In K. W. Spence and J. T. Spence (Eds.), The psychology of learning and motivation. New York: Academic Press, 1967.

AMSEL, A., HUG, J., \& SURRIDGE, C. Goal approaches and the partial reinforcement effect after minimal acquisition. Journal of Experimental Psychology, 1968, 77, 530-534. AMSEL, A., SURRIDGE, C., \& HUG, J. Number of food pellets and the partial reinforcement extinction effect after extended acquisition. Journal of Experimental Psychology, 1969, 82, 578-580.

LOGAN, F. A. Incentive. New Haven: Yale University Press, 1960.
MeCAIN. Ci. Difterent levels of performance with equivalent weights of reward. P'sychonomic Science, 1969, 14, 2-3

PUBOLS, B. H. Incentive magnitude, leaming, and perfomance in animals. Psychological Bulletin, 1960, 57, 89-115.

SPENCE, K. W. Behavior theory and learning. Englewood Cliffs, N.J: Prentice-Hall, 1960.

THEIOS, J., \& BRELSFORD, J Overlearning-extinction effect as an incentive phenomenon. Joumal of Experimental Psychology, 1964, 67, 463-467.

TRAPOLD, M. A., \& BELL, J. E. Effect of noncontingent exposure to shifts in reward magnitude on subsequent instrumental runway performance. Psychological Reports, 1964, 15, 679-684.

TRAPOLD, M. A., \& DOREN, D. G. Effect of noncontingent partial reinforcement on the resistance to extinction of a runway response. Journal of Experimental Psychology, 1966, $71,429-431$.

\section{NOTES}

1. Note that this argument does not necessarily apply to partial reinforcement training, since here persistence training may enhance resistance to extinction above that attributable to $r_{R}$.

2 . The last two blocks of five extinction trials were omitted from analysis since several Ss had reached the criterion for extinction, leaving some groups with too few $S$ s for reliable analysis.

\title{
Different nocturnal activity patterns of Peromyscus californicus and Peromyscus cremicus in lunar lighting
}

\author{
DONALD H. OWINGS and ROBERT B. LOCKARD \\ University of Washington, Seattle, Wash. 98105
}

The total night-time activity-wheel running by two sympatric species of mice, $P$. californicus and $P$. eremicus, was compared under the same simulated sun and moon cycles. Total running by $P$. californicus correlated negatively with the nightly duration of moonlight, while that of $P$. eremicus correlated positively. The results were discussed in terms of a temporal dimension of competitive exclusion.

The time of day at which a species is most active is ecologically significant. Most squirrels (family Sciuridae) have morphological, physiological, and behavioral adaptations for activity during sunlight, whereas most desert rodents are active at night, presumably an adaptation reducing dessication and predation. Many examples of much more precise timing of activities are known: the rising of the palolo worm (Clark \& Hess, 1942), the synchrony of grunion spawning with tides (Walker, 1952), and the rhythm of emergence of Drosophila from pupae (e.g., Pittendrigh \& Bruce, 1957) synchronized with the beginning of day in this diumal animal. Thus there may be certain times of day or conditions during a day for which activity has been selected. Although the adaptive features are partly understood in many such instances, they are not understood in others, and the phenomena remain tentatively labeled "species differences" until analysis of the ecological significance provides an understanding of the selective advantage of the timing of the activity.

Among the many probable ecological factors relevant to activity, one could be temporal competitive exclusion. The competitive exclusion principle (e.g., Hardin, 1960) states that two species cannot occupy the same niche in the same way because one of them will be the more successful, be it ever so slight an advantage, will increase in proportion, and will eventually occupy the niche exclusively. The usual interpretation of this principle is spatial-that sympatric species are in somewhat different habitats within the area of overlap. It is possible, however, that the dimension in which lack of competition evolved can also be temporal. Two species active at different times during the 24-h day would not exploit the environment in exactly the same way and could therefore theoretically coexist. 


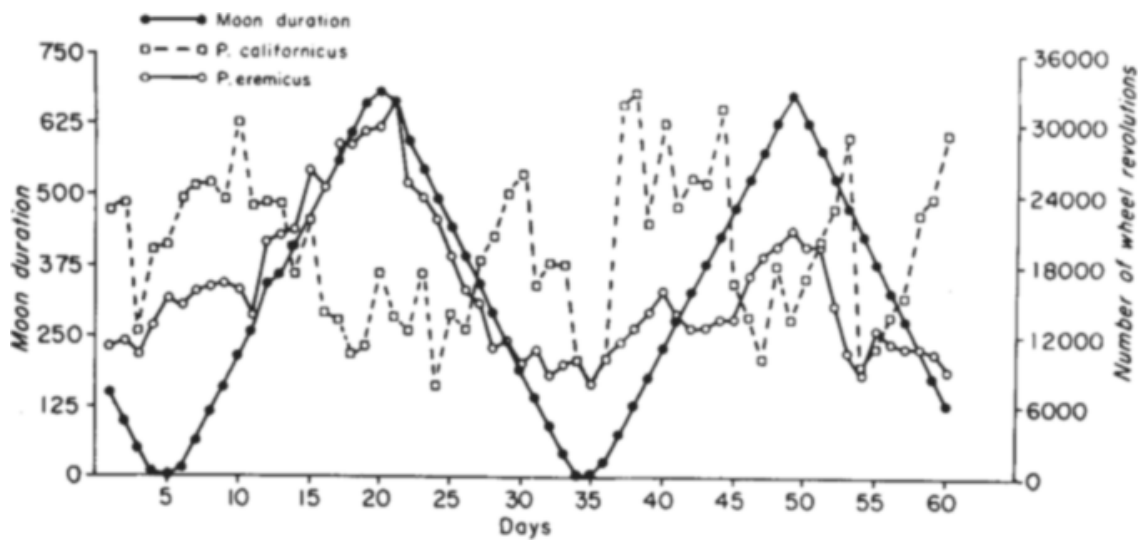

Fig. 1. Group total nightly activity wheel revolutions for $P$. californicus $(\mathrm{N}=8)$ and $P$. eremicus $(\mathbf{N}=7)$, and nightly moon duration (in minutes) as a function of time.

Two species of deer mice, Peromyscus californicus and Peromyscus eremicus (the California mouse and the cactus mouse) both feed on seeds, vegatation, invertebrates, and larvae; and both are often found interspersed among the traps of the same trap-line on brushy slopes in San Diego County, California. They are quite similar in morphology and ecological requirements, and on the basis of what is known one might expect direct competition between them; yet the niche overlap must not be complete because neither has displaced the other.

The purpose of this study was to test for a possible temporal competitive exclusion between these two mouse species in view of the temporal interpretation which may follow from the competitive exclusion principle and in view of the probable lack of other excluding factors.

\section{SUBJECTS}

Seven Peromyscus eremicus ( 400,3 oq) and eight $P$. californicus ( $4 \delta^{\circ}, 4 \%$ \%) were trapped on a sparsely brushy west slope of Cowles Mountain approximately 5 miles west and 1 mile south of Santee, San Diego County, California. They were air-freighted to the laboratory in Seattle where they were maintained for 4 months in the 12:12-hour day-night cycle later used in the experiment. At least the last 27 days of this habituation period was in the activity-wheel units used in the experiment. These were Wahmann LC-34 units with living cage plus a 3-in.-diam, 4-in. Jong nestbox. Each wheel unit was inside a $3 / 4-$ in. plywood compartment with an individual "sun" (Sylvania F8T5/W fluorescent lamp producing about $10 \mathrm{fc}$ ) and a "moon" (GE No. 39 incandescent lamp producing about $.01 \mathrm{fc}$ ). The moon was programmed to be on for $12 \mathrm{~h}$ and off for $12 \mathrm{~h} 50 \mathrm{~min}$, closely approximating the natural lunar lighting schedule.

The temporal pattern of activity for each animal was recorded on an Esterline-Angus event recorder. Wheel revolutions were recorded nightly on two counters for each species, one for revolutions while the moon was off, the other while the moon was on.

\section{RESULTS}

The nightly duration of moonlight and the nightly running-wheel activity of each species is shown in Fig. 1 for two complete lunar cycles. The apparent tendency of $P$. eremicus to be most active on moonlight nights is supported by the high correlation between activity and moonlight duration $(\mathrm{r}=.73, \mathrm{df}=58, \mathrm{p}<.005)$. Conversely, $P$. californicus showed the opposite relationship; it tended to be least active on moonlight nights $(\mathrm{r}=-.33, \mathrm{df}=58$, $\mathrm{p}<.01)$.

\section{DISCUSSION}

The results are in accord with the hypothesis that similar species occupying the same habitat and competing for similar food may show a temporal activity separation. The results are also consonant with certain known differences in the biology of the two species. $P$. californicus is larger in size and has smaller litters than eremicus (Rood, 1966), and tends toward a strong pair bond. Theoretically, a large mouse should have a history of more severe selection pressure by predators because of its easier visual detection and greater caloric reward per unit hunt.

The response to such selection could be a return to small size or toward behavioral adaptations which reduced predation by canceling the disadvantages of large size. At least the target size type of disadvantage would be reduced by confining surface activity to the darkest portions of the night. Evidence for the successful evolution of behaviors resulting in low predation is the low reproductive rate and the pair bond. A social system characterized by pair bonds implies some advantageous role of the male and would seem unlikely to evolve when mortality was high and the continued existence of the male was improbable. In contrast, the small size, higher reproductive rate, and unpaired social system of eremicus is consistent with the general strategy of a more risky existence with higher mortality.

These results, however, should be regarded as tentative, for a number of important questions remain unanswered. It is not known how these mice behave temporally in their habitat, as opposed to the laboratory, and this is the only criterion of importance to evolutionary processes. Furthermore, the developmental pathways leading to the temporally separated nocturnal activity suggested by the results are unknown; the same two species from a habitat of dense cover might behave differently. Also, the role of $P$. californicus in interspecific aggression is unknown; because it is the larger, it may dominate the habitat while it is active, forcing $P$. eremicus into the temporally less desirable niche. If this were the case, the two mice species would behave differently in the parts of their ranges where they are not sympatric. Temporal exclusion alone is not sufficient to eliminate competition; there must be specific aspects of resource utilization in which lack of competition leads to temporal exclusion. These specific aspects remain to be identified. While these possible complications may at first seem unwelcome in that they are impediments to generalizations, they also serve to illustrate just the opposite: that as we begin to understand how ecological factors act as selection pressures producing adaptive behaviors, we also begin to understand how to formulate a better kind of predictive paradigm than the practice of casually extending a finding to other species in other ecological situations.

\section{REFERENCES}

CLARK, L. B., \& HESS, W. N. Swarming of the A tlantic palolo worm, Leodice fucata (Ehlers). Papers from Tortugas Laboratory, 1942, 33, 21-70. Carnegie Institute of Washington Publication 524.

HARDIN, G. The competitive exclusion principle. Science, 1960, 131, 1292-1297.

PITTENDRIGH, C. S., \& BRUCE, V. G. An oscillator model for biological clocks. In D. Rudnick (Ed.), Rhythmic and synthetic processes in growth. Princeton: Princeton University Press, 1957. Pp. 75-109.

ROOD, J. P. Observations on the reproduction of Peromyscus in captivity. The American Midland Naturalist, 1966, 76, 496-503.

WALKER, B. W. A guide to the grunion. California Fish and Game, 1952, 38, 409-420. 